\title{
Revue
}

\section{Optimisation des soins chez les personnes âgées gravement malades}

\author{
Olivia Geen MD, Bram Rochwerg MD MSc, Xuyi Mimi Wang MD MÉdM
}

- Citation : CMAJ 2021 October 4;193:E1525-33. doi : 10.1503/cmaj.210652-f

Voir la version anglaise de l'article ici : www.cmaj.ca/lookup/doi/10.1503/cmaj.210652

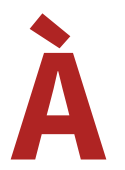

mesure que la population mondiale vieillit et que les progrès de la médecine permettent aux gens de maintenir une bonne qualité de vie à 80,90 ou même 100 ans, on note une augmentation des hospitalisations d'adultes de 65 ans et plus (personnes âgées) dans les unités de soins intensifs (USI). Les personnes âgées représentent maintenant plus de $50 \%$ des patients des USI au Canada ${ }^{1}$. À ce jour, durant la pandémie de COVID-19, $59,3 \%$ des patients atteints de COVID-19 hospitalisés dans une USI au Canada avaient plus de 60 ans $^{2}$. Les personnes qui doivent séjourner dans une USI sont jugées " gravement malades ». Les personnes âgées gravement malades constituent une population particulière qui a des besoins de soins distincts en raison des phénomènes physiologiques et pathologiques liés à l'âge. Nous passons ici en revue les pratiques thérapeutiques optimales chez les personnes âgées gravement malades, sur la base des données probantes disponibles (encadré 1 ). Nous discutons de l'intégration des principes gériatriques fondés sur des données probantes aux soins intensifs, de l'importance de détecter la fragilité préexistante chez les personnes âgées, des mesures pour prévenir et traiter le délirium et de l'optimisation des soins post-USI.

\section{Pourquoi et comment devrait-on intégrer les principes gériatriques fondés sur des données probantes aux soins habituels des USI?}

Étant donné que les personnes âgées sont plus susceptibles de présenter des besoins médicaux et sociaux complexes, il est important d'utiliser une approche thérapeutique holistique et globale, indépendamment du milieu de soins. Le nombre croissant de personnes âgées nécessitant des soins intensifs a mené à la naissance d'un nouveau domaine : les soins intensifs gériatriques. Les gériatres sont des experts des soins aux personnes âgées fragiles, et la communauté intensiviste s'intéresse de plus en plus à l'adoption des principes gériatriques fondés sur des données probantes dans les USI ${ }^{3-11}$. Les gériatres participent déjà à des modèles de collaboration

\section{Points clés}

- L'intégration des principes gériatriques aux soins habituels dans les unités des soins intensifs (USI) devrait faire partie des soins globaux pour toutes les personnes âgées.

- La fragilité est associée de manière indépendante à un risque plus élevé de décès à l'USI et doit être analysée dès l'admission à l'aide de l'échelle de fragilité clinique en fonction de l'état clinique du patient au moins 2 semaines avant l'épisode de maladie aiguë.

- Les mesures d'intervention groupées ABCDEF devraient être utilisés pour aider à prévenir et traiter le délirium chez les personnes âgées hospitalisées dans une USI.

- Le syndrome post-soins intensifs s'accompagne notamment de l'apparition ou de l'aggravation de déficits cognitifs, physiques ou mentaux, qui peuvent persister après le congé de l'USI.

- Les survivants âgés d'un séjour à l'USI pourraient bénéficier de services de réadaptation physique, d'un carnet de suivi pour l'USI et de l'intégration des principes gériatriques aux soins habituels post-USI.

\section{Encadré 1 : Interrogation de la littérature}

Nous avons procédé à une interrogation ciblée et non systématique de la base MEDLINE (1946 à février 2020) à l'aide des mots clés " geriatrics » et " critical care » ou « intensive care ». La recherche a été limitée aux articles de langue anglaise. Il n'y avait aucune limite quant au type d'étude, mais nous avons privilégié les essais randomisés et contrôlés, les revues systématiques et les méta-analyses portant sur le sujet à l'étude. Les études pertinentes ont été sélectionnées et leurs listes bibliographiques ont été traitées manuellement pour recenser d'autres articles. La plupart des études étaient observationnelles.

efficaces avec les orthopédistes, les cardiologues, les chirurgiens vasculaires, les traumatologistes et les oncologues, et on dispose de données probantes sur les bienfaits de ces modèles pour les patients ${ }^{10,12-15}$. Il y a toutefois un important obstacle aux modèles de soins en collaboration au Canada, soit la 
relative rareté des gériatres: on en compte moins de 400 au pays. Il est donc plus pratique de recourir à d'autres moyens pour intégrer les principes de l'évaluation gériatrique globale aux soins habituels à l'USI.

L'intégration des principes gériatriques aux soins habituels a été bien étudiée dans des unités de soins gériatriques de courte durée (USGCD) spécialisées pour les personnes âgées hospitalisées qui présentent un problème de santé aigu, mais qui n'ont pas besoin d'être admises aux soins intensifs. Dans une revue systématique et méta-analyse de 13 essais regroupant 6839 patients âgés en moyenne de 81 ans, l'application des principes gériatriques dans les USGCD a permis de réduire le déclin fonctionnel iatrogène, les chutes, le délirium, la durée du séjour hospitalier, les coûts et les transferts vers des établissements de soins de longue durée ${ }^{16}$. Le tableau 1 résume les interventions en plusieurs volets généralement utilisées dans les USGCD. Les modèles de soins en collaboration visant le développement des capacités et l'approfondissement des compétences gériatriques du personnel des USI, de même que les stratégies novatrices comme les évaluations gériatriques brèves à partir des dossiers médicaux électroniques ${ }^{25}$, sont des domaines prometteurs pour la recherche à venir.

Les soins globaux restent incomplets si l'on ne tient pas compte du milieu de vie des patients. Les gériatres utilisent une approche biopsychosociale pour planifier les soins. Même si les données actuelles sont de qualité très faible à modérée, les lignes directrices sont favorables à une participation plus grande des familles à l'USI (présence, soutien, communication $)^{26}$. On peut trouver en ligne des ressources sur les soins centrés sur la famille (https://www.sccm.org/Research/ Guidelines/Guidelines/Family-Centered-Care-in-the-ICU).

\section{Pourquoi tenir compte de la fragilité préexistante des personnes âgées gravement malades?}

La prévalence de la fragilité chez les patients âgés hospitalisés dans les USI est d'environ $30 \%$, selon les résultats regroupés de 6 études observationnelles prospectives ${ }^{27}$. La fragilité est un état de déclin des capacités physiologiques, fonctionnelles et cognitives qui aggrave la vulnérabilité à de nouveaux stresseurs médi$\operatorname{cau}^{28}$. On croit qu'elle découle de l'interaction des comorbidités et des facteurs génétiques et environnementaux ${ }^{29}$, et au stade intermédiaire, elle pourrait être en partie réversible ${ }^{30}$. La fragilité ne fait pas nécessairement partie du vieillissement, même si l'âge est un facteur de risque à cet égard, en plus d'être un facteur de risque indépendant de complications ${ }^{31}$.

À l'USI, il convient de vérifier la fragilité dans le cadre d'une évaluation globale pour mieux comprendre le risque de complications chez un patient et guider les discussions sur les objectifs des soins ${ }^{29,32-35}$. Dans une étude de cohorte multicentrique prospective sur 610 patients de plus de 80 ans au Canada, on a découvert que la fragilité était un prédicteur indépendant de complications à long terme des suites d'un séjour dans une USI plus significatif que l'âge, la gravité de la maladie et les comorbidités ${ }^{36}$.
Une méta-analyse de 2017 regroupant 10 études de cohorte prospectives de qualité modérée (score de Newcastle-Ottawa moyen 6,5 ) a révélé que la fragilité préhospitalière était associée à une hausse de la mortalité hospitalière (risque relatif [RR] 1,71, intervalle de confiance [IC] à $95 \% 1,43-2,05)$ et de la mortalité à long terme (RR 1,53, IC à $95 \%$ 1,40-1,68), indépendamment de l'âge ou de la gravité de la maladie ${ }^{27}$. Plusieurs études ont signalé une augmentation de la mortalité à chaque point additionnel à l'échelle de fragilité clinique $(\mathrm{EFC})^{27,33,37,38}$, particulièrement chez les individus présentant une grande ou une très grande fragilité $(E F C \geq 7)^{31,38}$. Comprendre l'incidence de la fragilité sur le pronostic à l'USI fait que l'on s'intéresse moins à l'âge du patient et plus à son état clinique global et à sa trajectoire avant son admission à l'USI.

En ce qui concerne la morbidité post-USI, les patients fragiles qui survivent à un épisode de maladie aiguë présentent un déclin du fonctionnement physique et un taux de placement en établissement de soins de longue durée plus importants que les personnes âgées qui ne sont pas fragiles ${ }^{27,37,39,40}$. Une étude longitudinale prospective de 15 ans sur 754 adultes de plus de 70 ans vivant dans la communauté a révélé que les patients en état de préfragilité (présence de 1 ou 2 critères de fragilité selon le modèle phénotypique de Fried) ou de fragilité ( 3 critères ou plus) n'avaient pas retrouvé leur fonctionnement physique de départ après 6 mois $^{39}$. Par contre, ils présentaient une amélioration par rapport à leur fonctionnement 1 mois après leur congé de l'USI, alors que l'atteinte était à son maximum ${ }^{39}$. En comparaison, les personnes âgées jugées non fragiles à leur admission à l'USI avaient pratiquement retrouvé leur fonctionnement physique de départ en l'espace de 6 mois $^{39}$. Les patients jugés fragiles avaient un taux de placement en établissement de soins de longue durée de $58,8 \%$ après 6 mois $^{39}$. Cette observation concorde avec celles d'une méta-analyse de 2017 selon laquelle ces patients étaient moins susceptibles de pouvoir retourner à la maison $^{27}$ (RR 0,59, IC à $95 \% 0,49-0,71$ ). Lors des discussions avec les patients et les familles de leurs attentes post-USI, le fait de mentionner explicitement la fragilité les empêche de surestimer l'atteinte fonctionnelle chez ceux qui ne sont pas fragiles, ou de la sous-estimer chez ceux qui sont fragiles (figure 1). On ignore par contre si les complications post-USI sont inévitables chez les patients fragiles puisqu'aucune étude n'a vérifié en quoi les changements de prise en charge durant ou après le séjour à l'USI permettent de réduire l'incidence ou de prévenir l'aggravation des complications associées à la fragilité. Il s'agit d'un domaine important pour la recherche future.

Les 2 principaux cadres conceptuels d'évaluation de la fragilité sont le modèle physiologique, ou phénotypique ${ }^{41}$, et le modèle (d'accumulation) des déficits, ou indice de fragilité ${ }^{28}$. On peut utiliser plusieurs outils inspirés de ces cadres pour mesurer la fragilité ${ }^{42}$. Chez les personnes âgées admises dans une USI, nous favorisons l'échelle de fragilité clinique présentée à la figure 2. L'EFC est en étroite corrélation avec l'indice de fragilité ${ }^{28}$ et sa validité repose sur une bonne fiabilité interexaminateurs $(\kappa 0,74)$ dans le contexte des USI ${ }^{43-46}$. Son utilisation est plus pratique chez les patients gravement malades que celle d'autres outils d'usage courant qui requièrent une 
Tableau 1 : Liste de vérification globale en plusieurs volets pour les soins habituels à l'USI*

\begin{tabular}{|c|c|}
\hline Principe & Suggestions pour la pratique courante \\
\hline Prévention du délirium & $\begin{array}{l}\text { - Port des prothèses auditives et des lunettes } \\
\text { - Application de mesures groupées ABCDEF† } \\
\text { - Utilisation minimale des contentions }\end{array}$ \\
\hline Sommeil & $\begin{array}{l}\text { - Bouchons d'oreille, réduction du bruit } \\
\text { - Passage à l'alimentation par bolus durant le jour pour réduire les interruptions nocturnes } \dagger\end{array}$ \\
\hline Cognition & - Activités de stimulation cognitive telles que musique, réorientation induite par une voix familière et participation de la famille \\
\hline Humeur & $\begin{array}{l}\text { - Dépistage des symptômes dépressifs chez les patients dont le séjour à l'USI est prolongé et consultation en psychiatrie au besoin } \\
\text { - Dépistage non suggéré chez les patients récemment admis présentant un problème aigu† }\end{array}$ \\
\hline $\begin{array}{l}\text { Mobilité et réadaptation } \\
\text { hâtives }\end{array}$ & $\begin{array}{l}\text { - Évaluation hâtive en physiothérapie ou en ergothérapie pour améliorer la mobilité et le fonctionnement pour le maintien des } \\
\text { activités de la vie quotidienne }\end{array}$ \\
\hline Nutrition & $\begin{array}{l}\text { - Consultation en diététique } \\
\text { - Correction rapide de la déshydratation }\end{array}$ \\
\hline Continence & $\begin{array}{l}\text { - Retrait des cathéters à demeure pour éviter les infections urinaires liées à la sonde et promouvoir la mobilité } \\
\text { - Maintien de la régularité intestinale }\end{array}$ \\
\hline Intégrité cutanée & - Changements fréquents de position pour éviter les plaies de lit \\
\hline $\begin{array}{l}\text { Réduction de la } \\
\text { polypharmacie }\end{array}$ & $\begin{array}{l}\text { - Revue quotidienne des médicaments par le pharmacien au moyen des critères STOPP/START }{ }^{17} \text { ou des critères BEERS }{ }^{18} \text { de l'American } \\
\text { - Seriatrics Society, pour repérer tout médicament potentiellement inapproprié } \\
\text { d'arrêt durant le séjour à l'USI† }\end{array}$ \\
\hline $\begin{array}{l}\text { Modifications de } \\
\text { l'environnement pour faciliter } \\
\text { le fonctionnement physique et } \\
\text { cognitif }\end{array}$ & $\begin{array}{l}\text { - Horloges et calendriers de grande taille } \\
\text { - Rampes et couloirs dégagés pour faciliter la mobilité } \\
\text { - Surélévation des sièges de toilette et poignées de porte à levier (plutôt que rondes) } \\
\text { - Couleurs de peinture aux tons de terre, et contraste entre plancher, murs et plafond pour aider les patients qui ont des problèmes } \\
\text { de perception de la profondeur }\end{array}$ \\
\hline Planification précoce du congé & $\begin{array}{l}\text { - Inclusion hâtive du service de travail social et de la famille } \\
\text { - Réunion de l'équipe multidisciplinaire et insistance sur l’objectif du retour à la maison (ou au milieu de vie préhospitalier) }\end{array}$ \\
\hline
\end{tabular}

Remarque : START = Screening Tool to Alert to Right Treatment, STOPP = Screening Tool of Older Persons' Prescriptions, USGCD = unité de soins gériatriques de courte durée, USI = unité de soins intensifs.

*Selon les principes fondés sur des données probantes pour les USGCD ${ }^{19-23}$

†Non inclus dans les protocoles traditionnels des USGCD. Les mesures groupées ABCDEF constituent une stratégie multiple pour la prévention et le traitement du délirium et incluent la gestion de la douleur, les essais d'éveil spontané, le choix des analgésiques et de la sédation, la surveillance et la prise en charge du délirium, la mobilisation précoce et la participation des familles ${ }^{24}$.

évaluation de la force préhensile ou de la mobilité, par exemple $^{41,47}$. Pour éviter de surévaluer le score à l'EFC en se fiant à l'état d'un patient gravement malade à l'USI, l'examen devrait se fonder sur l'état clinique au moins 2 semaines avant l'admission ${ }^{48}$. Si on dispose de renseignements insuffisants de la part du patient ou de ses proches pour effectuer cette évaluation, on peut trouver des informations collatérales auprès d'intervenants communautaires, comme les préposés aux services de soutien, les pharmaciens, les médecins de famille et les coordonnateurs de soins communautaires locaux. On encourage les médecins qui connaissent peu l'EFC à se renseigner sur son utilisation appropriée pour assurer la fiabilité des résultats ${ }^{48,49}$.

\section{Pourquoi est-il important de reconnaître le délírium?}

Le délirium est un trouble fréquent, mais sous diagnostiqué, dans les USI, avec une prévalence allant de $20 \%$ à $84 \%$ selon la gravité de la maladie et la méthode diagnostique utilisée ${ }^{50-55}$. II se définit par une altération abrupte et variable de l'attention et de l'état de conscience, et il s'accompagne de dysfonctions cognitives (perte de mémoire, désorientation, propos décousus, dérèglement des aptitudes ou de la perception visuospatiales) ${ }^{56}$.

Une méta-analyse de 2015 a révélé que le délirium à l'USI est associé à une mortalité accrue (RR 2,19, IC à $95 \%$ 1,78-2,70) une observation qui persistait même après une métarégression pour tenir compte de l'âge, de la proportion de participants de sexe féminin et des scores APACHE II (Acute Physiology and Chronic Health Evaluation II) -, de même qu'à une durée prolongée de la ventilation mécanique (différence moyenne [DM] 1,79 jour de plus), du séjour à l'USI (DM 33 heures de plus) et du séjour hospitalier (DM 23,3 heures de plus) et à une atteinte cognitive persistant 3 et 12 mois après le congé, comparativement aux patients qui ne présentaient pas de délirium ${ }^{51}$.

Les facteurs de risque de délirium à l'USI sont multiples : utilisation de benzodiazépines, transfusions sanguines, âge avancé, antécédents de démence, antécédents de coma, scores APACHE II et de l'American Society of Anesthesiology élevés et 


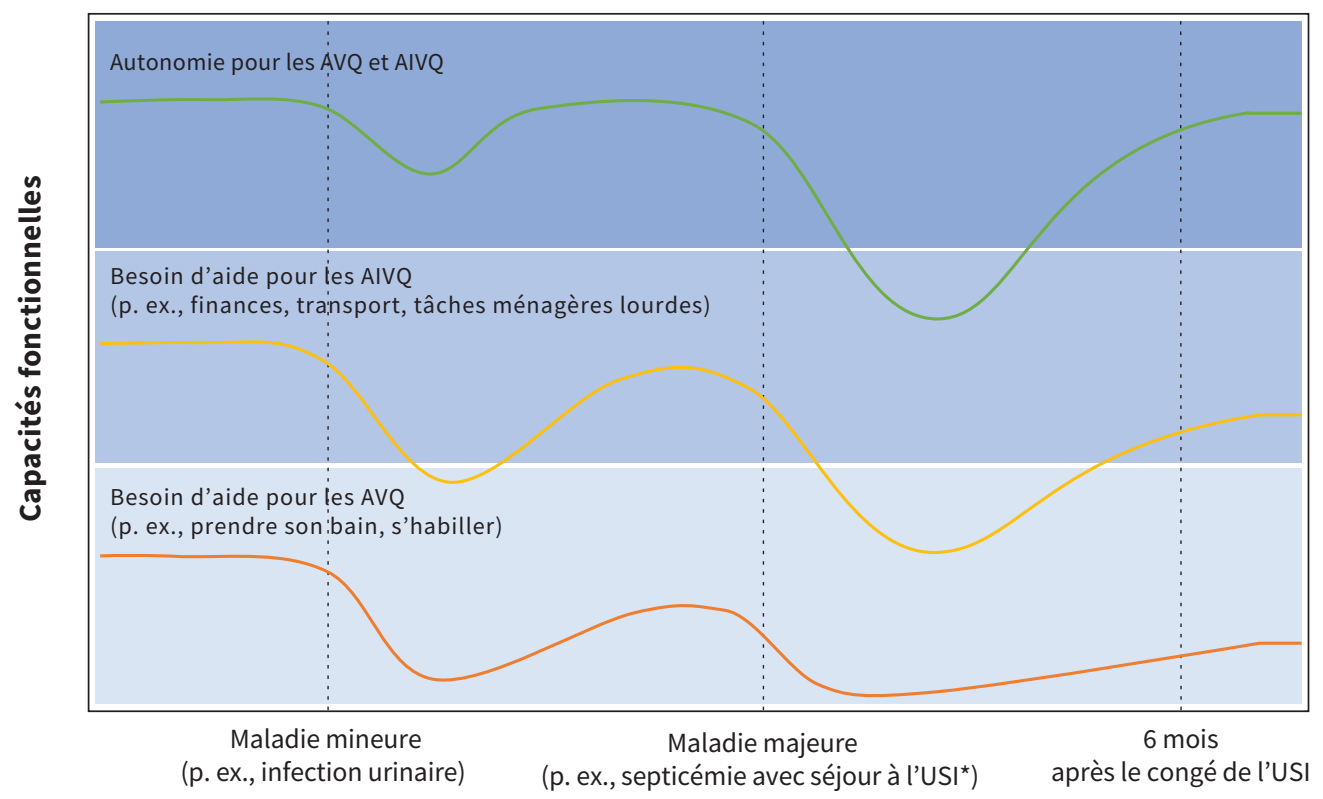

Chronologie

\begin{abstract}
Figure 1 : Incidence potentielle de la fragilité préexistante sur les complications d'une maladie mineure et majeure. La ligne verte représente le parcours médical d'une personne non fragile (1-3 sur l'échelle de fragilité clinique [EFC], indépendamment des activités et activités instrumentales de la vie quotidienne [AVQ et AIVQ]) : une maladie mineure peut causer un déclin transitoire du fonctionnement physique ou cognitif, mais la personne retrouve ses capacités de départ. Une maladie majeure nécessitant l'admission dans une unité de soins intensifs peut causer un déclin substantiel du fonctionnement et des AVQ, mais un patient qui n'est pas fragile pourra retrouver son fonctionnement de départ ou presque en l'espace de 6 mois. La ligne jaune représente le parcours médical d'une personne présentant une légère fragilité (4-5 sur l'EFC) : une maladie mineure peut occasionner un déclin disproportionné du fonctionnement et la personne pourrait ne pas retrouver ses pleines capacités de départ. Une maladie majeure qui nécessite l'admission dans une unité de soins intensifs pourrait entraîner un déclin substantiel du fonctionnement et la personne ne se rétablira que partiellement dans les 6 mois suivants. La ligne orange représente le parcours médical d'une personne présentant une fragilité de modérée à grave (6-8 sur l'EFC) : une maladie mineure risque de causer un déclin disproportionné du fonctionnement déjà limité, sans retour aux capacités de départ, et une maladie majeure risque d'entraîner un déclin substantiel du fonctionnement qui ne s'améliorera pas dans les 6 mois, en supposant que la personne survive à sa maladie (la mortalité hospitalière lorsque l'EFC est à 8 serait de $48 \%{ }^{31}$, et la survie à 12 mois lorsque l'EFC est à 6-7, de $\left.35 \%{ }^{37}\right)$. Remarque : USI = unité de soins intensifs.
\end{abstract}

chirurgie urgente ou traumatisme avant le séjour à l'USI ${ }^{57}$. Parmi ces éléments, les benzodiazépines sont un facteur potentiellement modifiable ${ }^{58,59}$; cette classe de médicaments est à éviter à moins d'indications précises pour un problème de santé précis, comme un sevrage aigu d'alcool.

\section{Comment peut-on prévenir et gérer le délirium?}

Étant donné que le délirium passe souvent inaperçu, il est important de savoir mieux le reconnaître. Les lignes directrices de pratique clinique PADIS (Pain, Agitation/Sedation, Delirium, Immobility and Sleep Disruption) de 2018 recommandent le dépistage du délirium au moyen d'un outil valide ${ }^{57}$, comme les outils CAMICU (Confusion Assessment method for the ICU) ${ }^{60}$ et ICDSC (Intensive Care Delirium Screening Checklist) ${ }^{61}$. L'ICDSC a une sensibilité de $99 \%$ et une spécificité de $64 \%{ }^{61}$, et la CAM-ICU a une sensibilité de $75,5 \%$ et une spécificité de 95,8\% ${ }^{60}$.

Les interventions non pharmacologiques forment la base de la prévention du délirium à l'USI. Une méta-analyse de 9 études a révélé que les bouchons d'oreille réduisaient l'incidence du délirium (RR 0,59, IC à $95 \% 0,44-0,78)^{62}$, ce qui en fait une intervention envisageable, inoffensive et peu coûteuse. Plusieurs petits essais randomisés et contrôlés (ERC) d'une généralisabilité limitée se sont penchés sur d'autres interventions uniques et ont obtenu des résultats négatifs; ces interventions comprenaient la thérapie cognitive $e^{63}$, la réorientation induite par une voix familière ${ }^{64}$ et la luminothérapie ${ }^{65}$.

Les données sont plus solides avec les interventions multiples ${ }^{24,66-68}$, ce qui indique que le délirium est un problème multifactoriel. Les lignes directrices PADIS font état d'un rapport de cotes (RC) de 0,59 (IC à $95 \% 0,39-0,88$ ) pour la baisse de l'incidence du délirium lors de l'utilisation de mesures d'intervention groupées ${ }^{57}$. Les mesures groupées $A B C D E F$ constituent un cadre opérationnalisé de ces lignes directrices; leurs éléments incluent : gestion de la douleur, essai d'éveil spontané, choix d'analgésie et de sédation, surveillance et gestion des épisodes de délirium, mobilisation hâtive et implication de la famille ${ }^{24}$. Les principes qui 


\section{ÉCHELLE DE FRAGILITÉ CLINIQUE}

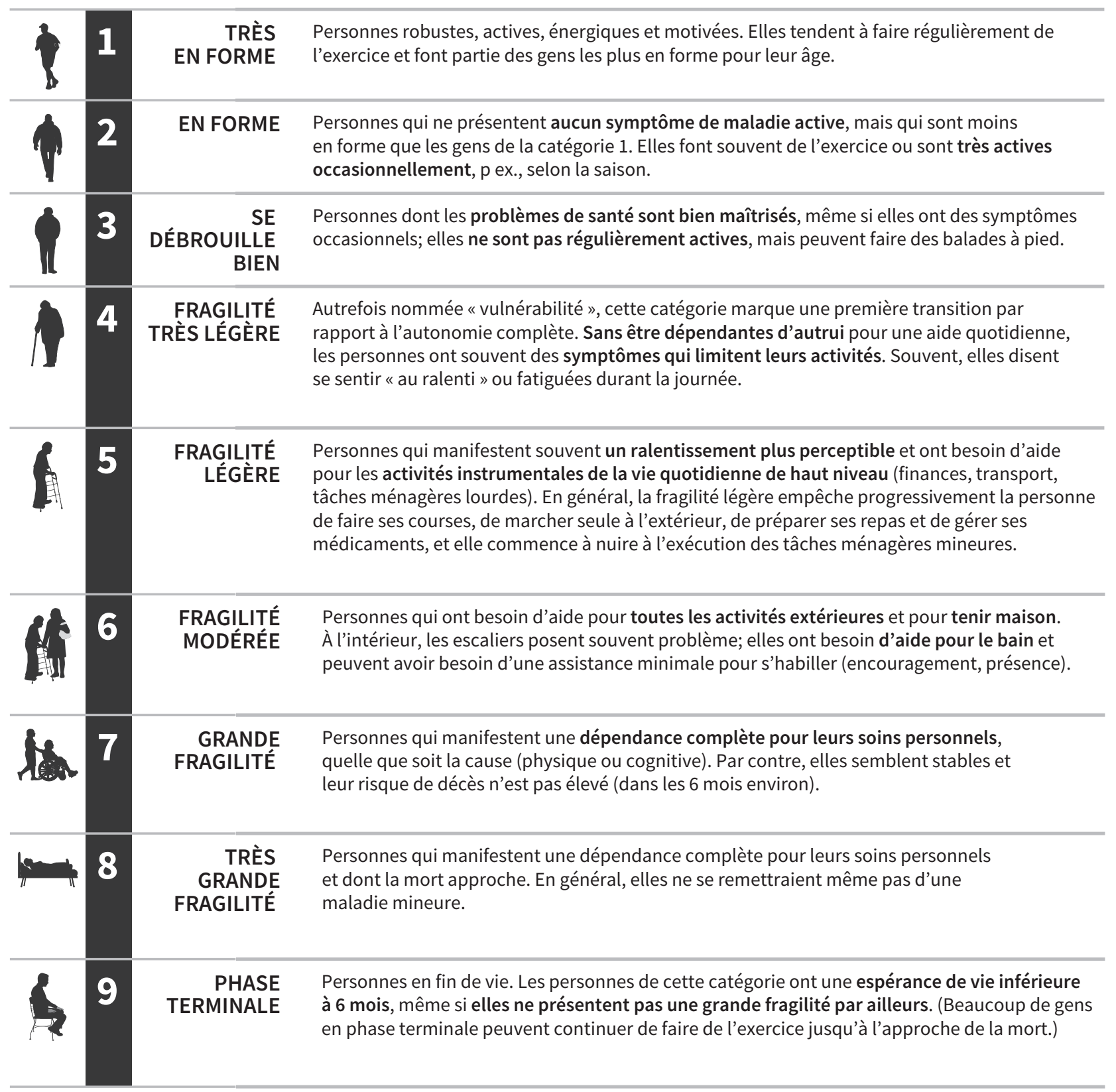

\section{SCORES DE FRAGILITÉ CHEZ LES PERSONNES ATTEINTES DE DÉMENCE}

Le degré de fragilité correspond généralement au degré de démence. Les symptômes courants de la démence légère incluent: oublier les détails d'un événement récent, sans oublier l'événement lui-même, répéter les mêmes questions et histoires, et se replier sur soi.
Dans la démence modérée, la mémoire récente est très affectée même si les personnes semblent bien se souvenir d'événements de leur passé. Les personnes peuvent voir à leurs soins personnels avec des encouragements.

Dans la démence grave, les personnes ne peuvent pas voir à leurs soins personnels sans aide.

Dans la démence très grave, les personnes sont souvent confinées au lit. Plusieurs ne parlent pour ainsi dire plus.

Figure 2 : L'échelle de fragilité clinique (EFC) peut être utilisée pour faire le point sur l'état clinique global d'un patient en fonction de ses comorbidités, de son degré d'activité et de son déclin fonctionnel. Lors de conversations avec le patient, ses proches ou d'autres personnes concernées, on a recours au jugement clinique pour déterminer à quelle catégorie le patient appartient. On recommande de baser le score sur l'état du patient 2 semaines avant son admission à l'unité de soins intensifs (USI) [reproduit avec l'autorisation de Rockwood et al. ${ }^{28}$ ]. 
sous-tendent les mesures $A B C D E F$ recoupent la démédicalisation et les principes centrés sur le patient de multiples interventions déjà utilisées dans les USGCD (résumées au tableau 1). Dans une volumineuse étude de cohorte multicentrique prospective sur plus de 15000 patients, l'utilisation des interventions groupées a donné lieu à une réduction proportionnelle à la dose de l'incidence du délirium ( $\mathrm{RC} 0,60$, IC à $95 \%$ 0,49-0,72), du coma, du recours aux contentions, des réadmissions à l'USI et des réadmissions et de la mortalité à l'hôpital ${ }^{24}$. Les futures études devraient s'attarder à des stratégies de mise en œuvre et d'application des connaissances; des ressources pour la mise en $œ u v r e$ sont disponibles en ligne (www.icudelirium.org/ medical-professionals/overview).

Les antipsychotiques sont déconseillés pour prévenir les épisodes de délirium ${ }^{57}$, une méta-analyse Cochrane ${ }^{50}$ et un vaste ERC subséquent sur plus de 1700 patients n'ayant montré aucun avantage par rapport au placebo ${ }^{69}$. Chez les patients qui ont besoin d'une sédation pour d'autres indications et qui présentent un risque élevé de délirium, on peut envisager plutôt la dexmédétomidine. Même si les lignes directrices PADIS déconseillent l'utilisation de la dexmédétomidine pour la prévention du délirium ${ }^{57}, 2$ revues systématiques et métaanalyses récentes suggèrent qu'elle est associée à une baisse de l'incidence de ce trouble $e^{70,71}$.

La plupart des données appuyant l'utilisation de mesures groupées concernent la prévention du délirium, mais ces mesures sont aussi recommandées pour le traitement étant donné que leurs avantages potentiels en surclassent les risques $^{24,57}$. Les antipsychotiques ne sont pas efficaces, comme l'a montré I'ERC multicentrique MIND USA (Modifying the Impact of ICU-Associated Neurological Dysfunction-USA) mené sur 1789 patients : il n'y a eu aucune différence d'effet entre l'halopéridol, la ziprasidone et le placebo pour ce qui est de la durée des épisodes de délirium ${ }^{54}$. Les lignes directrices PADIS appuient l'utilisation de la dexmédétomidine lorsque l'agitation associée au délirium empêche le sevrage ou l'extubation ${ }^{57}$, selon un seul ERC de faible qualité ${ }^{72}$. L'efficacité de la dexmédétomidine pour le délirium sans agitation reste incertaine, et on suggère une réduction des doses chez les sujets de plus de 65 ans, en raison des taux élevés de bradycardie et d'hypotension ${ }^{73}$.

Lorsque les symptômes de délirium, comme les hallucinations, l'anxiété ou l'agitation, nuisent à la santé psychologique ou physique des patients ou posent un risque pour la santé des travailleurs de la santé, un antipsychotique pourrait être nécessaire. Le cas échéant, il est préférable de suivre le principe gériatrique qui consiste à "y aller petit à petit » et à en prescrire un à court terme et au besoin pour éviter son utilisation involontaire après le départ de l'USI ou de l'hôpital. Une étude de cohorte observationnelle prospective a révélé que $24 \%$ des patients traités pour délirium au moyen d'un antipsychotique atypique avaient reçu leur congé de l'hôpital avec ce médicament ${ }^{74}$. La remise d'une telle ordonnance au moment du congé résulte probablement de l'inertie et peut entraîner l'utilisation prolongée du médicament.

\section{Quelles complications post-USI les médecins devraient-ils prévoir chez les personnes âgées qui survivent à un épisode de maladie grave?}

Grâce aux progrès médicaux et technologiques, la mortalité associée aux séjours à l'USI a diminué et la plupart des personnes âgées survivent désormais à une maladie grave. Parmi les survivants d'un séjour à l'USI âgés de plus de 80 ans, les taux de mortalité à long terme à 1,2 et 3 ans après le congé hospitalier sont comparables à ceux de la population générale assortie selon l'âge et le sexe ${ }^{75}$. Une étude prospective sur 3920 patients âgés en moyenne de 84 ans provenant de 22 pays a révélé un taux de survie de $72,5 \%$ après un séjour à l'USI, et un taux de survie de $61,2 \%$ à 30 jours $^{34}$. Par contre, survivre à un épisode de maladie grave peut s'accompagner d'une morbidité et d'un déclin fonctionnel à long terme associés au séjour à l'USI, 2 issues importantes à prévoir et à gérer de manière proactive après le séjour.

On ne connaît pas bien la prévalence du syndrome post-soins intensifs (SPSI) chez les adultes, mais il affecterait de $25 \%$ à $55 \%$ ou plus des survivants d'un séjour à l'USI ${ }^{76,77}$. Le syndrome englobe un groupe hétérogène d'atteintes cognitives, physiques ou mentales nouvelles ou exacerbées ${ }^{78}$ (figure 3), qui peuvent inclure le trouble de stress post-traumatique (TSPT) ( $44 \%$ à 6 mois) ${ }^{79}$, un déclin des activités instrumentales de la vie quotidienne ${ }^{80}$, la dépression ( $34 \%$ à 6 mois $^{81}$ ) et une atteinte cognitive (34\% à 12 mois $^{82}$ ). Étant donné l'éventail des atteintes, les patients qui présentent un SPSI pourraient avoir besoin d'un niveau supérieur de soins ou de soutien par un aidant naturel après le congé hospitalier. Le SPSI - famille, un groupe similaire de complications observées chez les proches de survivants d'un séjour à l'USI, inclut le TSPT, nouveau ou exacerbé, la dépression et une situation de deuil ou d'anxiété compliquée ${ }^{83}$.

La gestion du SPSI après le congé est un domaine où les connaissances progressent. Pour la plupart des patients qui reçoivent leur congé de l'hôpital, le suivi et la réadaptation par des spécialistes font défaut, la polypharmacie est courante et les soins sont fragmentés ${ }^{84}$. Plusieurs revues systématiques se sont penchées sur diverses interventions de suivi post-USI ${ }^{85-88}$, mais leurs résultats sont difficiles à interpréter étant donné la faible certitude des données et l'hétérogénéité des populations, des interventions, des contextes (hospitalier et extra-hospitalier) et des paramètres mesurés. Nous avons trouvé 5 études contrôlées au cours desquelles le groupe soumis à l'intervention était en moyenne âgé de plus de $65 \mathrm{ans}^{89-93}$. Aucune étude n'a fourni d'analyses de sous-groupes selon le degré de fragilité, une limite majeure compte tenu que la fragilité préexistante influence substantiellement l'issue. D'autres études sur la gestion, après le congé, des personnes âgées ayant survécu à un séjour à l'USI sont requises, avec une stratification a priori des sous-groupes selon le degré de fragilité. Malgré ces limites, certaines observations peuvent s'appliquer à ces personnes.

Une grande étude de cohorte basée dans la population à Taiwan regroupant plus de 15000 survivants d'une septicémie âgés en moyenne de 69,4 ans a révélé que la réadaptation physique dans les 90 jours suivant le congé de l'USI a donné lieu à 


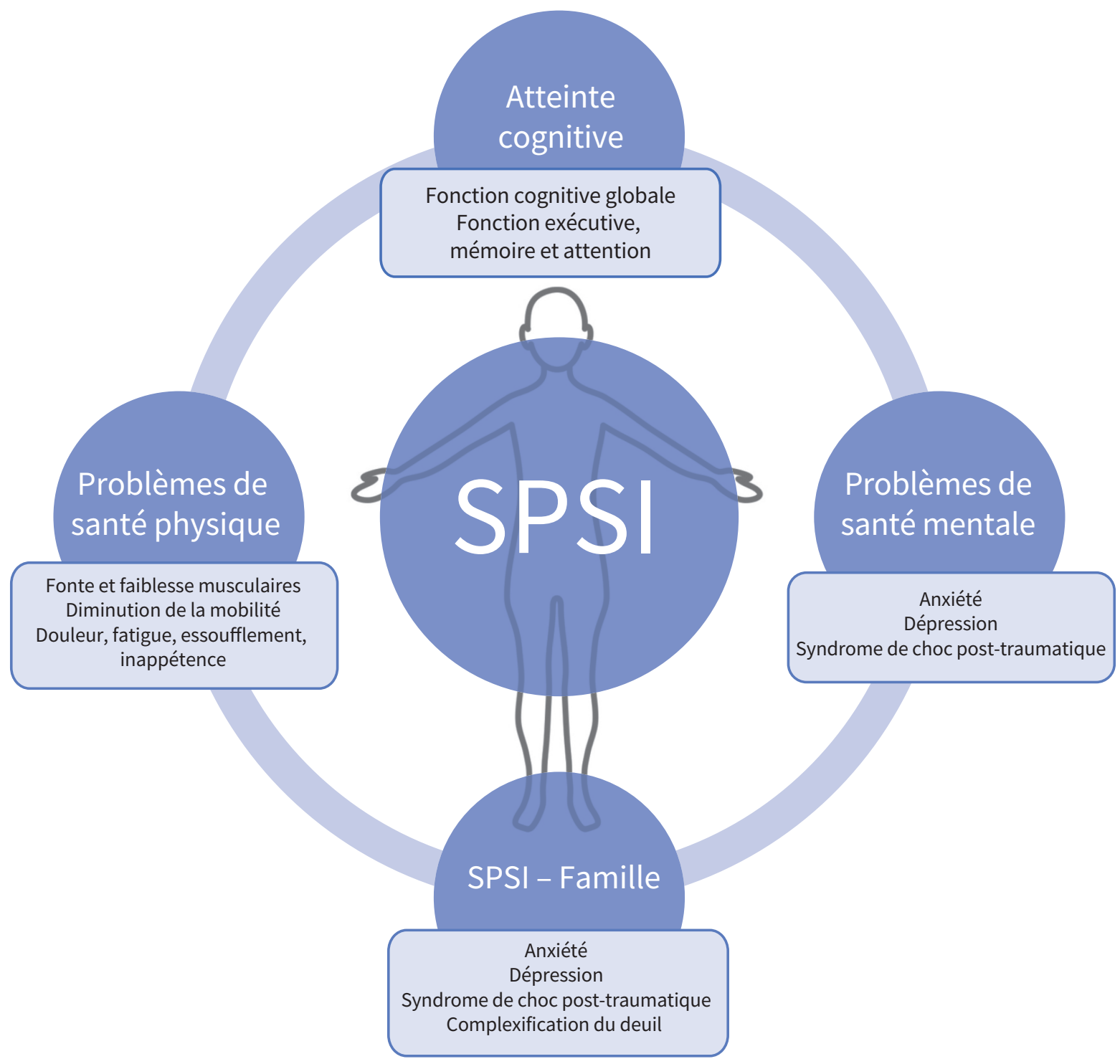

Figure 3 : Éléments du syndrome post-soins intensifs (SPSI). Les survivants d'un séjour à l'unité des soins intensifs (USI) peuvent présenter des atteintes cognitives, physiques et mentales. Les proches peuvent aussi éprouver des problèmes de santé mentale après le séjour d'un être cher à l'USI.

une baisse de $8 \%$ de la mortalité à un an (risque relatif [RR] 0,92, IC à $95 \%$ 0,88-0,96) et à une réduction de 5,6\% de la mortalité à 10 ans (RR 0,94, IC à $95 \% 0,92-0,97)^{90}$. La mortalité a diminué proportionnellement au nombre de séances de réadaptation dont les participants ont bénéficié ${ }^{90}$. Une récente revue systématique de 16 ERC et de 10 études observationnelles sur des adultes ayant survécu à un séjour à l'USI a également révélé le bienfait de la physiothérapie. On y a observé que les interventions axées sur les capacités physiques amélioraient la dépression et la qualité de vie liée à la santé mentale ${ }^{85}$. À noter qu'il semble que les avantages soient moindres si la population n'est pas correctement ciblée, ce qui pourrait expliquer en partie pourquoi certaines études sur la réadaptation n'ont pas conclu aux mêmes bienfaits dans d'autres populations ${ }^{94-96}$. Par exemple, l'étude de Taiwan a montré qu'il n'y avait aucun avantage sur la survie chez les patients dont le séjour à l'USI ou la durée de la ventilation mécanique était de moins de 7 jours, ou chez ceux qui présentaient moins de comorbidités (indice de comorbidité de Charlson $\leq 2)^{90}$. Cela laisse entendre que les patients les plus susceptibles de bénéficier d'une réadaptation physique sont ceux qui sont moins bien au départ ou qui ont une maladie grave prolongée et qui, par conséquent, courent un risque accru de fonte musculaire et de déconditionnement. Déterminer quelles interventions fonctionnent, pour qui, et dans quelles conditions aidera les équipes soignantes à éviter la sur- et la sous-utilisation des ressources pour les soins post-USI centrés sur le patient. Une synthèse réaliste (qui utilise une approche systématique pour comprendre les mécanismes qui sous-tendent les résultats d'une intervention) des mesures post-USI est en cours et orientera, nous l'espérons, la voie à suivre en matière de soins post-USI ${ }^{97}$.

D'autres interventions potentiellement efficaces chez les personnes âgées comprennent le recours à un carnet de suivi d'USI92 
et l'intégration des principes de l'USGCD aux soins post-USI89. Un carnet de suivi permet à la famille et à l'équipe soignante de prendre des notes durant le séjour d'un patient à l'USI afin d'éviter les oublis ${ }^{92}$. Dans la population générale des USI, selon 2 revues systématiques, un tel carnet a été associé à un risque moindre de dépression et à une meilleure qualité de vie ${ }^{98,99}$. Les principes des SGCD se sont montrés prometteurs dans un petit ERC en France qui, malgré sa puissance statistique insuffisante, a révélé une tendance à l'amélioration de l'autonomie fonctionnelle lorsque les personnes âgées étaient admises à une unité gériatrique appliquant les principes de l'USGCD après le congé de l'USI, comparativement aux soins habituels d'un service de médecine ${ }^{89}$. Beaucoup de politiques hospitalières appuient déjà l'intégration des principes gériatriques dans les soins habituels pour tous les patients âgés sous la forme d'initiatives de soins adaptés à l'âge ${ }^{100-103}$. L'intégration des principes de SGCD résumés au tableau 1 peut être envisagée pour les survivants d'un séjour à I'USI admis dans un service de médecine ou de chirurgie afin de prévenir le déclin iatrogène des capacités. On dispose de plusieurs études sur les innovations systématiques et technologiques qui facilitent l'application des principes de SGCD ${ }^{104-107}$.

Durant un séjour hospitalier ou peu après le congé, une demande de consultation en gériatrie pour une atteinte cognitive, en psychiatrie gériatrique pour des problèmes de santé mentale et en physiatrie pour l'optimisation du fonctionnement physique pourrait se révéler utile en présence de certains éléments spécifiques du SPSI, même si les stratégies de demandes de consultation n'ont pas été directement étudiées. D'autres stratégies de prise en charge post-congé, telles que des services infirmiers de suivi post-USI seraient inefficaces selon les données actuelles ${ }^{86,87}$.

\section{Conclusion}

Les conseils sur la meilleure façon de soigner les personnes âgées gravement malades sont limités en raison d'un manque d'ERC portant spécifiquement sur cette population et d'études qui stratifient les résultats selon le degré de fragilité. Malgré ces limites, nous avons recensé certaines options pour améliorer les soins, y compris l'évaluation de la fragilité préexistante comme outil pronostique dans les USI, l'utilisation d'interventions non pharmacologiques multiples pour prévenir et traiter les épisodes de délirium, l'intégration des principes gériatriques aux soins de routine à l'USI et la prise en compte de la forte prévalence des atteintes cognitives, physiques et mentales consécutives à un séjour dans une USI. La participation des gériatres, qui sont des experts de la fragilité, de l'atteinte cognitive et des soins aux personnes âgées, pourrait aider les intensivistes et les hospitalistes à se concentrer sur la nature aiguë des soins intensifs et postintensifs, et à répondre aux besoins particuliers des personnes âgées. La recherche future devra porter entre autres sur les modèles de soins gériatriques en collaboration dans les USI, l'application des principes gériatriques pendant la période postUSI et l'évaluation de la fragilité péri-USI afin de créer et d'appliquer des plans de traitement individualisés et adaptés à la trajectoire médicale globale des patients (encadré 2).

\section{Encadré 2 : Questions sans réponse}

- Quelles autres interventions durant ou après une admission à l'unité des soins intensifs (USI) permettent de prévenir l'incidence ou l'aggravation de la fragilité?

- Comment la fragilité influence-t-elle la réussite des interventions utilisées pour gérer le syndrome post-soins intensifs?

- Les modèles de soins en collaboration qui utilisent les principes gériatriques sont-ils efficaces pour la prise en charge des personnes âgées gravement malades durant ou après un séjour à l'USI, et quels éléments sont nécessaires à leur succès?

\section{Références}

1. Care in Canadian ICUs: data tables. Ottawa: Canadian Institute for Health Information. Accessible ici : https://www.cihi.ca/en/care-in-canadian-icus -data-tables (consulté le 18 août 2021).

2. COVID 19 daily epidemiology update. Ottawa: Public Health Agency of Canada; updated 2021 Sept. 3. Accessible ici : https://health-infobase.canada.ca/src/ data/covidLive/Epidemiological-summary-of-COVID-19-cases-in-Canada-Canada .ca.pdf (consulté le 15 sept. 2021).

3. Joyce MF, Reich JA. Critical care issues of the geriatric patient. Anesthesiol Clin 2015;33:551-61.

4. Flaatten $\mathrm{H}$, de Lange DW, Artigas $\mathrm{A}$, et al. The status of intensive care medicine research and a future agenda for very old patients in the ICU. Intensive Care Med 2017;43:1319-28.

5. Michels G, Sieber CC, Gernot M, et al. Geriatric intensive care: Consensus paper of DGIIN, DIVI, DGAI, DGGG, ÖGGG, ÖGIAIN, DGP, DGEM, DGD, DGNI, DGIM, DGKliPha and DGG [article en allemand]. Z Gerontol Geriatr 2019;52:440-56.

6. Conroy S, Parker S. Acute care for frail older people: time to get back to basics? Age Ageing 2014;43:448-9.

7. Brummel NE, Ferrante LE. Integrating geriatric principles into critical care medicine: the time is now. Ann Am Thorac Soc 2018;15:518-22.

8. Hao J-F, Cui H-M, Han J-M, et al. Tele-ICU: The way forward in geriatric care? Aging Clin Exp Res 2014;26:575-82.

9. Guidet B, Vallet H, Boddaert J, et al. Caring for the critically ill patients over 80 : a narrative review. Ann Intensive Care 2018;8:114.

10. Damluji AA, Forman DE, van Diepen S, et al.; American Heart Association Council on Clinical Cardiology and Council on Cardiovascular and Stroke Nursing. Older adults in the cardiac intensive care unit: factoring geriatric syndromes in the management, prognosis, and process of care: a scientific statement from the American Heart Association. Circulation 2020;141:e6-32.

11. Tardini F, Pinciroli R, Berra L. The intensive care unit: how to make this unfriendly environment more geriatric-friendly. Eur J Surg Oncol 2020; 46:379-82.

12. Grigoryan KV, Javedan H, Rudolph JL. Orthogeriatric care models and outcomes in hip fracture patients: a systematic review and meta-analysis. $J$ Orthop Trauma 2014;28:e49-55.

13. Partridge JSL, Harari D, Martin FC, et al. Randomized clinical trial of comprehensive geriatric assessment and optimization in vascular surgery. Br J Surg 2017;104:679-87.

14. Eagles D, Godwin B, Cheng W, et al. A systematic review and meta-analysis evaluating geriatric consultation on older trauma patients. J Trauma Acute Care Surg 2020;88:446-53.

15. Kalsi T, Babic-Illman G, Ross PJ, et al. The impact of comprehensive geriatric assessment interventions on tolerance to chemotherapy in older people. $\mathrm{Br} \mathrm{J}$ Cancer 2015;112:1435-44.

16. Fox MT, Persaud M, Maimets I, et al. Effectiveness of acute geriatric unit care using acute care for elders components: a systematic review and metaanalysis. J Am Geriatr Soc 2012;60:2237-45.

17. O'Mahony D, O'Sullivan D, Byrne S, et al. STOPP/START criteria for potentially inappropriate prescribing in older people: version 2. Age Ageing 2015;44:213-8.

18. 2019 American Geriatrics Society Beers Criteria Updated Expert Panel. American Geriatrics Society 2019 updated AGS Beers Criteria for potentially inappropriate medication use in older adults. J Am Geriatr Soc 2019;67:674-94.

19. Counsell SR, Holder CM, Liebenauer LL, et al. Effects of a multicomponent intervention on functional outcomes and process of care in hospitalized older patients: a randomized controlled trial of Acute Care for Elders (ACE) in a community hospital. J Am Geriatr Soc 2000;48:1572-81. 
20. Palmer RM. The acute care for elders unit model of care. Geriatrics (Basel) 2018;3:59.

21. Landefeld CS, Palmer RM, Kresevic DM, et al. A randomized trial of care in a hospital medical unit especially designed to improve the functional outcomes of acutely ill older patients. N Engl J Med 1995;332:1338-44.

22. Barnes DE, Palmer RM, Kresevic DM, et al. Acute care for elders units produced shorter hospital lengths of stays at lower costs while maintaining patients' functional status. Health Aff (Millwood) 2012;31:1227-36.

23. Flood KL, Booth K, Vickers J. Acute Care for Elders (ACE) team model of care: a clinical overview. Geriatrics (Basel) 2018;3:50.

24. Pun BT, Balas MC, Barnes-Daly MA, et al. Caring for critically ill patients with the ABCEDEF Bundle: results of the ICU liberation collaborative in over 15,000 adults. Crit Care Med 2019;47:3-14.

25. Katlic MR, Coleman J, Khan K, et al. Sinai abbreviated geriatric evaluation: development and validation of a practical test. Ann Surg 2019;269:177-83.

26. Davidson JE, Aslakson RA, Long AC, et al. Guidelines for family-centered care in the neonatal, pediatric, and adult ICU. Crit Care Med 2017;45:103-28.

27. Muscedere J, Waters B, Varambally A, et al. The impact of frailty on intensive care unit outcomes: a systematic review and meta-analysis. Intensive Care Med 2017;43:1105-22.

28. Rockwood K, Song X, Macknight C, et al. A global clinical measure of fitness and frailty in elderly people. CMAJ 2005;173:489-95.

29. McDermid RC, Stelfox HT, Bagshaw SM. Frailty in the critically ill: a novel concept. Crit Care 2011;15:301.

30. Negm AM, Kennedy CC, Thabane L, et al. Management of frailty: a systematic review and meta-analysis of randomized controlled trials. J Am Med Dir Assoc 2019;20:1190-8.

31. Darvall JN, Bellomo R, Bailey M, et al. Frailty and outcomes from pneumonia in critical illness: a population-based cohort study. Br J Anaesth 2020;125:730-8.

32. Flaatten H, De Lange DW, Morandi A, et al.; VIP1 study group. The impact of frailty on ICU and 30-day mortality and the level of care in very elderly patients (> 80 years). Intensive Care Med 2017;43:1820-8.

33. De Geer L, Fredrikson M, Tibblin AO. Frailty predicts 30-day mortality in intensive care patients: a prospective prediction study. Eur J Anaesthesiol 2020;37:1058-65.

34. Guidet B, de Lange DW, Boumendil A, et al.; VIP1 study group. he contribution of frailty, cognition, activity of daily life and comorbidities on outcome in acutely admitted patients over 80 years in European ICUs; the VIP2 study. Intensive Care Med 2020;46:57-69.

35. De Biasio JC, Mittel AM, Mueller AL, et al. Frailty in critical care medicine: a review. Anesth Analg 2020;130:1462-73.

36. Heyland DK, Garland A, Bagshaw S, et al. Recovery after critical illness in patients aged 80 years or older: a multi-center prospective observational cohort study. Intensive Care Med 2015;41:1911-20.

37. Brummel NE, Bell SP, Girard TD, et al. Frailty and subsequent disability and mortality among patients with critical illness. Am J Respir Crit Care Med 2017;196:64-72.

38. Hendin A, Tanuseputro P, Mclsaac D, et al. Frailty is associated with decreased time spent at home after critical illness: a population-based study. J Intensive Care Med 2021;36:937-44

39. Ferrante LE, Pisani MA, Murphy T, et al. The association of frailty with post-ICU disability, nursing home admission, and mortality. Chest 2018;153:1378-86.

40. Bagshaw SM, Stelfox HT, McDermid RC, et al. Association between frailty and short-and long-term outcomes among critically ill patients: a multicentre prospective cohort study. CMAJ 2014;186:E95-102.

41. Fried LP, Tangen CM, Walston J, et al.; Cardiovascular Health Study Collaborative Research Group. Frailty in older adults: evidence for a phenotype. J Gerontol A Biol Sci Med Sci 2001;56:M146-56.

42. Buta BJ, Walston JD, Godino JG, et al. Frailty assessment instruments: systematic characterization of the uses and contexts of highly-cited instruments. Ageing Res Rev 2016;26:53-61.

43. Pugh RJ, Battle CE, Thorpe C, et al. Reliability of frailty assessment in the critically ill: a multicenter prospective observational study. Anaesthesia 2019;74:758-64.

44. Pugh RJ, Ellison A, Pye K, et al. Feasibility and reliability of frailty assessment in the critically ill: a systematic review. Crit Care 2018;22:49.

45. Shears M, Takaoka A, Rochwerg B, et al.; Canadian Critical Care Trials Group. Assessing frailty in the intensive care unit: a reliability and validity study. J Crit Care 2018;45:197-203.

46. Falvey JR, Ferrante LE. Frailty assessment in the ICU: translation to "realworld" clinical practice. Anaesthesia 2019;74:700-3.

47. Rolfson DB, Majumdar SR, Tsuyuki RT, et al. Validity and reliability of the Edmonton Frail Scale. Age Ageing 2006;35:526-9.

48. Rockwood K, Theou O. Using the Clinical Frailty Scale in allocating scarce health care resources. Can Geriatr J 2020;23:210-5.
49. Theou O, Pérez-Zepeda MU, van der Valk AM, et al. A classification tree to assist with routine scoring of the Clinical Frailty Scale. Age Ageing 2021; 50:1406-11.

50. Herling SF, Greve IE, Vasilevskis EE, et al. Interventions for preventing intensive care unit delirium in adults. Cochrane Database Syst Rev 2018;11:CD009783.

51. Salluh JIF, Wang H, Schneider EB, et al. Outcome of delirium in critically ill patients: systematic review and meta-analysis. BMJ 2015;350:h2538.

52. Garpestad E, Devlin JW. Polypharmacy and delirium in critically ill older adults; recognition and prevention. Clin Geriatr Med 2017;33:189-203.

53. Mehta S, Cook D, Devlin JW, et al. SLEAP Investigators; Canadian Critical Care Trials Group. Prevalence, risk factors, and outcomes of delirium in mechanically ventilated adults. Crit Care Med 2015;43:557-66.

54. Girard TD, Exline MC, Carson SS, et al.; MIND-USA Investigators. Haloperidol and Ziprasidone for treatment of delirium in critical illness. N Engl J Med 2018;379:2506-16.

55. Pandharipande PP, Ely EW, Arora RC, et al. The intensive care delirium research agenda: a multinational, interprofessional perspective. Intensive Care Med 2017;43:1329-39.

56. Diagnostic and statistical manual of mental disorders: DSM-5. 5th ed Washington (DC): American Psychiatric Association; 2013.

57. Devlin JW, Skrobik Y, Gélinas C, et al. Clinical practice guidelines for the prevention and management of pain, agitation/sedation, delirium, immobility, and sleep disruption in adult patients in the ICU. Crit Care Med 2018;46:e825-73.

58. Pandharipande $P$, Shintani A, Peterson J, et al. Lorazepam is an independent risk factor for transitioning to delirium in intensive care unit patients. Anesthesiology 2006;104:21-6.

59. Zaal IJ, Devlin JW, Hazelbag M, et al. Benzodiazepine-associated delirium in critically ill adults. Intensive Care Med 2015;41:2130-7.

60. Neto AS, Nassar AP Jr, Cardoso SO, et al. Delirium screening in critically ill patients: a systematic review and meta-analysis. Crit Care Med 2012; 40:1946-51.

61. Bergeron N, Dubois MJ, Dumont M, et al. Intensive care delirium screening checklist: evaluation of a new screening tool. Intensive Care Med 2001;27:859-64

62. Litton E, Carnegie V, Elliott R, et al. The efficacy of earplugs as a sleep hygiene strategy for reducing delirium in the ICU: a systematic review and metaanalysis. Crit Care Med 2016;44:992-9.

63. Brummel NE, Girard TD, Ely EW, et al. Feasibility and safety of early combined cognitive and physical therapy for critically ill medical and surgical patients: the Activity and Cognitive Therapy in ICU (ACT-ICU) trial. Intensive Care Med 2014;40:370-9.

64. Munro CL, Cairns P, Ji M, et al. Delirium prevention in critically ill adults through an automated reorientation intervention: a pilot randomized controlled trial. Heart Lung 2017;46:234-8.

65. Simons KS, Laheij RJF, van den Boogaard M, et al. Dynamic light application therapy to reduce the incidence and duration of delirium in intensive-care patients: a randomized controlled trial. Lancet Respir Med 2016;4:194-202.

66. Foster J, Kelly M. A pilot study to test the feasibility of a nonpharmacologic intervention for the prevention of delirium in the medical intensive care unit. Clin Nurse Spec 2013;27:231-8.

67. Moon K-J, Lee S-M. The effects of a tailored intensive care unit delirium prevention protocol: a randomized controlled trial. Int J Nurs Stud 2015; 52:1423-32.

68. Rivosecchi RM, Kane-Gill SL, Svec S, et al. The implementation of a nonpharmacologic protocol to prevent intensive care delirium. J Crit Care 2016;31:206-11.

69. van den Boogaard M, Slooter AJC, Brüggemann RJM, et al. Effect of haloperidol on survival among critically ill adults with a high risk of delirium: the REDUCE randomized clinical trial. JAMA 2018;319:680-90.

70. Ng KT, Shubash CJ, Chong JS. The effect of dexmedetomidine on delirium and agitation in patients in intensive care: systematic review and meta-analysis with trial sequential analysis. Anaesthesia 2019;74:380-92.

71. Pereira JV, Sanjanwala RM, Mohammed MK, et al. Dexmedetomidine versus propofol sedation in reducing delirium among older adults in the ICU: a systematic review and meta-analysis. Eur J Anaesthesiol 2020;37:121-31.

72. Reade MC, Eastwood GM, Bellomo R, et al. Effect of Dexmedetomidine added to standard care on ventilator-free time in patients with agitated delirium: a randomized clinical trial. JAMA 2016;315:1460-8

73. Product monograph: PrdexmedeTOMidine Hydrochloride for injection. Boucherville (QC): Sandoz Canada Inc.; revised 2020 Mar. 24. Accessible ici : https://pdf.hres.ca/dpd_pm/00056114.PDF (consulté le 17 oct. 2021).

74. Tomichek JE, Stollings JL, Pandharipande PP, et al. Antipsychotic prescribing patterns during and after critical illness: a prospective cohort study. Crit Care 2016;20:378. 
75. Atramont A, Lindecker-Cournil V, Rudant J, et al. Association of age with shortterm and long-term mortality among patients discharged from intensive care units in France. JAMA Netw Open 2019;2:e193215.

76. Ohtake PJ, Lee AC, Scott JC, et al. Physical impairments associated with postintensive care syndrome: systematic review based on the World Health Organization's international classification of functioning, disability and health framework. Phys Ther 2018;98:631-45.

77. Marra A, Pandharipande P, Girard T, et al. Co-occurrence of Post-Intensive Care Syndrome problems among 406 survivors of critical illness. Crit Care Med 2018;46:1392-1401.

78. Needham DM, Davidson J, Cohen H, et al. Improving long-term outcomes after discharge from intensive care unit: report from a stakeholders' conference. Crit Care Med 2012;40:502-9.

79. Parker AM, Sricharoenchai T, Raparla S, et al. Posttraumatic stress disorder in critical illness survivors: a metaanalysis. Crit Care Med 2015;43:1121-9.

80. Hopkins RO, Suchyta MR, Kamdar BB, et al. Instrumental activities of daily living after critical illness: a systematic review. Ann Am Thorac Soc 2017;14:1332-43.

81. Rabiee A, Nikayin S, Hashem MD, et al. Depressive symptoms after critical illness: a systematic review and meta-analysis. Crit Care Med 2016;44:1744-53.

82. Pandharipande PP, Girard TD, Jackson JC, et al. Long-term cognitive impairment following critical illness. N Engl J Med 2013;369:1306-16.

83. Cameron JI, Chu LM, Matte A, et al. One-year outcomes in caregivers of critically ill patients. N Engl J Med 2016;374:1831-41.

84. Brown SM, Bose S, Banner-Goodspeed V, et al.; Addressing Post Intensive Care Syndrome 01 (APICS-01) study team. Approaches to addressing post-intensive care syndrome among intensive care unit survivors: a narrative review. Ann Am Thorac Soc 2019;16:947-56.

85. Rosa RG, Ferreira GE, Viola TW, et al. Effects of post-ICU follow-up on subject outcomes: a systematic review and meta-analysis. J Crit Care 2019;52:115-25.

86. Jensen JF, Thomsen T, Overgaard D, et al. Impact of follow-up consultations for ICU survivors on post-ICU syndrome: a systematic review and metaanalysis. Intensive Care Med 2015;41:763-75.

87. Schofield-Robinson OJ, Lewis SR, Smith AF, et al. Follow-up services for improving long-term outcomes in intensive care unit (ICU) survivors. Cochrane Database Syst Rev 2018;11:CD012701.

88. Mehlhorn J, Freytag A, Schmidt K, et al. Rehabilitation interventions for post intensive care syndrome: a systematic review. Crit Care Med 2014;42:1263-71.

89. Somme D, Andrieux N, Guérot E, et al. Loss of autonomy among elderly patients after a stay in a medical intensive care unit (ICU): a randomized study of the benefit of transfer to a geriatric ward. Arch Gerontol Geriatr 2010;50:e36-40.

90. Chao P-w, Shih C-J, Lee Y-J, et al. Association of postdischarge rehabilitation with mortality in intensive care unit survivors of sepsis. Am J Respir Crit Care Med 2014;190:1003-11.

91. Salisbury LG, Merriweather JL, Walsh TS. The development and feasibility of a ward-based physiotherapy and nutritional rehabilitation package for people experiencing critical illness. Clin Rehabil 2010;24:489-500.

92. Garrouste-Orgeas M, Coquet I, Périer A, et al. Impact of an intensive care unit diary on psychological distress in patients and relatives. Crit Care Med 2012;40:2033-40.

93. Jensen JF, Egerod I, Bestle MH, et al. A recovery program to improve quality of life, sense of coherence and psychological health in ICU survivors: a multicenter randomized controlled trial, the RAPIT study. Intensive Care Med 2016;42:1733-43.

94. Cuthbertson BH, Rattray J, Campbell MK, et al.; PRaCTICaL study group. The PRaCTICaL study of nurse led, intensive care follow-up programmes for improving long term outcomes from critical illness: a pragmatic randomized controlled trial. BMJ 2009;339:b3723.

95. Connolly B, Thompson A, Douiri A, et al. Exercise-based rehabilitation after hospital discharge for survivors of critical illness with intensive care unitacquired weakness: a pilot feasibility trial. J Crit Care 2015;30:589-98.

96. Elliott D, McKinley S, Alison J, et al. Health-related quality of life and physical recovery after a critical illness: a multi-centre randomized controlled trial of a home-based physical rehabilitation program. Crit Care 2011;15:R142.
97. Howard AF, Currie L, Bungay V, et al. Health solutions to improve postintensive care outcomes: a realist review protocol. Syst Rev 2019;8:11.

98. Barreto BB, Luz M, de Oliveira Rios MN, et al. The impact of intensive care unit diaries on patients' and relatives' outcomes: a systematic review and metaanalysis. Crit Care 2019;23:411.

99. Mcllroy PA, King RS, Garrouste-Orgeas M, et al. The effect of ICU diaries on psychological outcomes and quality of life of survivors of critical illness and their relatives: a systematic review and meta-analysis. Crit Care Med 2019;47:273-9.

100. Fulmer T, Mate KS, Berman A. The age-friendly health system imperative. J Am Geriatr Soc 2018;66:22-4.

101. Conroy S. Silver Book: quality care for older people with urgent and emergency care needs. London (UK): British Geriatrics Society; 2012.

102. Horgan S. Designing integrated care for older adults living with complex and chronic health needs: a scoping review. Provincial Geriatrics Leadership Office; 2020.

103. Age-friendly health systems: guide to using the 4Ms in the care of older adults. Bonston: Institute for Healthcare Improvement; 2020.

104. Malone ML, Volbrecht M, Stephenson J, et al. Acute Care for Elders (ACE) tracker and e-Geriatrician: methods to disseminate ACE concepts to hospitals with no geriatricians on staff. J Am Geriatr Soc 2010;58:161-7.

105. Booth KA, Simmons EE, Viles AF, et al. Improving geriatric care processes on two medical-surgical acute care units: a pilot study. $J$ Healthc Qual 2019;41:23-31.

106. Hung WW, Ross JS, Farber J, et al. Evaluation of the Mobile Acute Care of the Elderly (MACE) service. JAMA Intern Med 2013;173:990-6.

107. Farber JI, Korc-Grodzicki B, Du Q, et al. Operational and quality outcomes of a mobile acute care for the elderly service. J Hosp Med 2011;6:358-63.

\section{Intérêts concurrents : Aucun déclaré.}

Cet article a été révisé par des pairs.

Affiliations : Division de médecine gériatrique (Geen, Wang) et de médecine de soins intensifs (Rochwerg), Départements de médecine et des méthodes, impacts et données probantes de la recherche en santé (Rochwerg), Université McMaster, Hamilton, Ont.

Collaborateurs : Olivia Geen et Mimi Wang ont contribué à la conception et à la modélisation du travail. Olivia Geen a contribué à l'acquisition des données. Tous les auteurs ont participé à l'analyse et à l'interprétation des données. Olivia Geen a rédigé le manuscrit. Bram Rochwerg et Mimi Wang l'ont révisé de façon critique pour tout contenu intellectuel important. Tous les auteurs ont donné leur approbation finale pour la version soumise pour publication et assument l'entière responsabilité de tous les aspects du travail.

Propriété intellectuelle du contenu : Il s'agit d'un article en libre accès distribué conformément aux modalités de la licence Creative Commons Attribution (CC BY-NC-ND 4.0), qui autorise l'utilisation, la diffusion et la reproduction dans tout médium à la condition que la publication originale soit adéquatement citée, que l'utilisation se fasse à des fins non commerciales (c.-à-d., recherche ou éducation) et qu'aucune modification ni adaptation n'y soit apportée. Voir : https://creativecommons.org/licenses/by -nc-nd/4.0/deed.fr.

Correspondance : Olivia Geen, olivia.geen@medportal.ca 